Original Research Paper

\title{
Novel Approach for Characterizing Solid Rocket Motor (SRM)
}

\author{
Aliyu Bhar Kisabo, Ngozi Nwokolo, Aliyu Funmilayo Adebimpe and Sholiyi Olusegun Samuel \\ Centre for Space Transport and Propulsion (CSTP) Epe, Lagos-Nigeria
}

Article history

Received: 06-02-2019

Revised: 01-03-2019

Accepted: 23-03-2019

Corresponding Author:

Aliyu Bhar Kisabo

Centre for Space Transport and

Propulsion (CSTP) Epe, Lagos-

Nigeria

Email: aliyukisabo@yahoo.com

\begin{abstract}
To characterize SRM, the motor needs to be tested on a static test rig and certain performers indicators evaluated. In this study, the thrust with time from the SRM was acquired via a Digital Acquisition (DAQ) System and saved in Microsoft Excel ${ }^{\circledR}$ format(.csv). These thrust and time of the tested motor were then imported into MATLAB ${ }^{\circledR}$ for the purpose of computing burn time, maximum thrust, average thrust, total impulse, specific impulse and chamber pressure of the motor. The specific impulse and combustion chamber pressure for the SRM were computed in a novel approach which we call Pseudo-numerical approach (combination of experimental result with numerical computation). In this approach, first, we modelled the depleting propellant mass in the combustion chamber with a novel mathematical expression. This novel expression was then used in computing specific impulse of the SRM. Second, the total surface area of the propellant grain was modelled as a hollow cylinder with depleting dynamics during motor operation, in a similar manner with the propellant mass. Third, multiplying the reciprocal of the propellant grain surface area with the experimental force gave use the pseudo-numerically computed chamber pressure. The pressure trend of the SRM obtained was also compared to those in well-established literature, thus, the pseudo-numerical approach put forward proves to be effective.
\end{abstract}

Keywords: Solid Rocket Motor (SRM), Thrust, Pressure, MATLAB ${ }^{\circledR}$

\section{Introduction}

The most widely used propulsion systems for applications that requires high thrust to weight ratio for relatively short intervals of time is the Solid rocket motors (Abdel et al., 2015). The word 'solid' in SRM connotes that the propellant used for the rocket motor is of the solid-state type.

A solid rocket motor is a rocket motor that uses a solid mixture of chemicals as its propellant. The Nozzle part of motor is responsible for thrust generation and experiences high amount of temperature and pressure changes during the motor operation. Both propellant and nozzle is crucial in the design and construction of rocket motor (Turner, 2006). A lot of work has been done on the pressure of SRM but none addresses the possibility of combining experimental thrust with numerical means of determining combustion chamber pressure. This method proposed in this study has the advantage of reducing the cost of experimental technique that will require extra sensors to characterize SRMs.
In this study, we will show by example how test result from SRM static test rig is used to determine certain performance criteria of the motor which will aid in the design of the rocket as a whole. Also, unveil the so-called novel pseudo-numerical means of determining chamber pressure. The experimental set-up to determine thrust for SEM consists basically of a load cell, analogue to digital converter and a Digital Acquisition system (DAQ) with a Graphical User Interface (GUI) installed on a laptop (Aliyu et al., 2015).

\section{Analyzing Experimental Results}

The DAQ system in the experimental set-up for thrust of SRM has a GUI wizard which when installed and calibrated properly will show graphically the thrust with time profile on the laptop. A better option to view this graph of thrust with time is to export these results into a .csv file from the DAQ wizard and re-plot the graph in a computational software like MATLAB. This option gives us the flexibility to call this type of file in 
MATLAB working environment and do other computations with ease aside giving a better plot than what the DAQ GUI. The .csv file for the time thrust data in this study was saved with the name Test_080518, after placing this file in working directory, the following code was used to load it into MATLAB working environment:

$$
x=\text { load ('Test_080518.csv') }
$$

Next, is to designate variables that will correspond to force and time associated with the $x$ above as follows:

$$
\begin{aligned}
& t=x(:, 1) \\
& f=x(:, 2)
\end{aligned}
$$

Now, to plot the result exactly as it was captured in the WinDaQ window for comparison with what MATLAB $^{\circledR}$ will produce is very necessary before any computation. The main reason for this is to ensure that the data imported into MATLAB ${ }^{\circledR}$ has not been distorted in any form. To this plotting, we simply used the command as $\operatorname{plot}(t, f)$ and it gave us what is depicted in Fig. 1. After a satisfactory visual comparison with the time against thrust profile as given in the WinDaQ GUI, we then proceeded with some editing of Fig. 1.

It is clearly seen that from Fig. 1, that there exists the need to zoom close to the region of activity, thus cuttingoff the regions that carries no information of use is necessary. From Fig. 1, we zoomed to realize Fig. 2. Using the following code:

$$
\begin{aligned}
& s t=f i n d(t==92) \\
& s p=f i n d(t==103)
\end{aligned}
$$

Notice that in Fig. 2, the maximum point attained by the thrust is about $1,519 \mathrm{~N}$ and observe very close that the thrust ( $y$-axis or thrust-axis) did not begin from zero (it started from about $246.2 \mathrm{~N}$ ). Thus, the profile in Fig. 2 needs to be brought to the zero point and the graduation on the Time axis ( $x$-axis) needs to be re-designated to also start from zero. The following codes were used to achieve the modifications shown in Fig. 3:

$$
\begin{aligned}
& t 1=t(\text { st:sp }) \\
& f 1=f(\text { st:sp }) \\
& f \_o f f s e t=f 1(1) \\
& t \text { offset }=t 1(1) \\
& f 2=f 1-f \text { offset } \\
& t 2=t 1-t \text { offset } \\
& \text { plot }(t 2, f 2)
\end{aligned}
$$

All necessary anomalies in Fig. 2 were corrected to give Fig. 3, from it, we can now compute our burnt time, deduce the maximum thrust, average thrust, total impulse and specific impulse and combustion chamber pressure of the SRM.

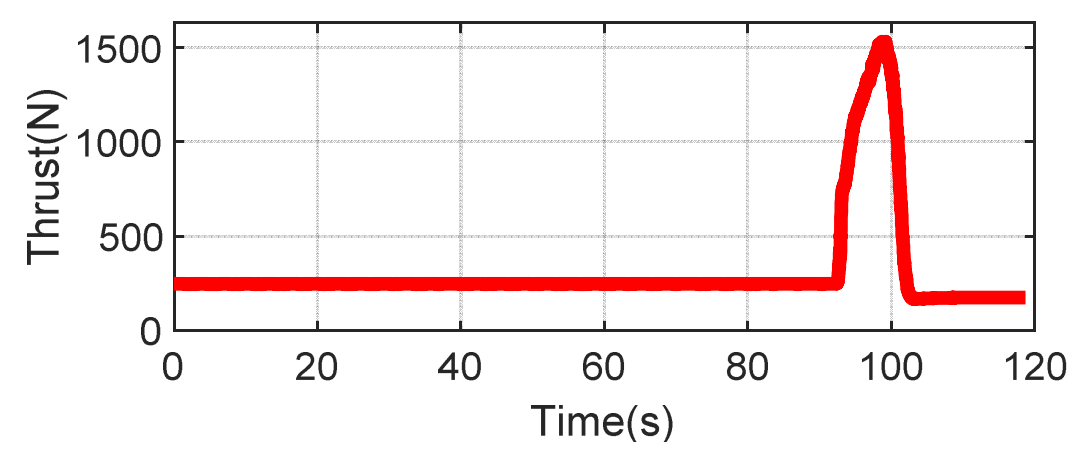

Fig. 1: Unaltered data imported from the WinDAQ

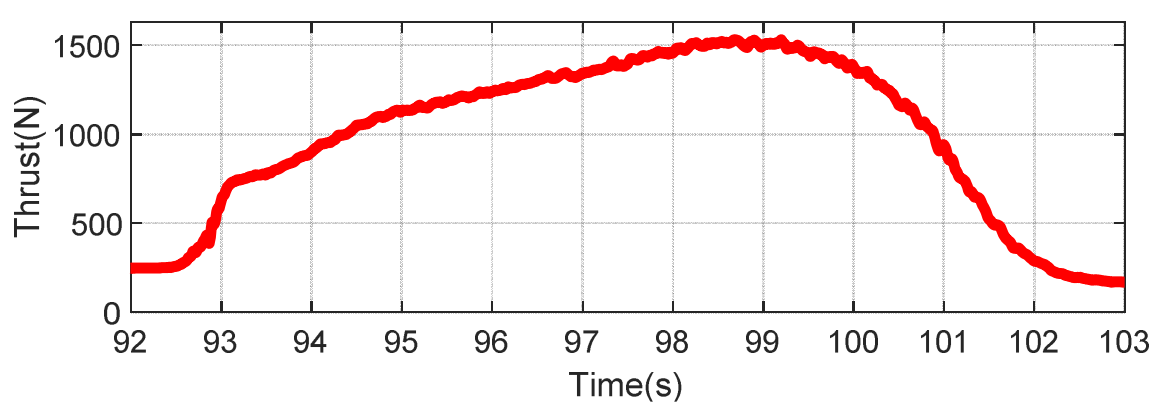

Fig. 2: Region of activity with marked out points 


\section{Burn Time}

Burn time is the number of seconds for which the motor produces thrust. This tells you how long the motor will keep pushing your rocket. (Burn time is determined by chopping off the ends of the curve when the thrust is below $5 \%$ of the maximum (MPDO, 2018). These points, $5 \%$ on both sides are depicted in Fig. 3. For burn time, with Fig. 3 in perspective, we deduce it by doing 10.03-0.74 $=9.29 \mathrm{~s}$. Notice that we could not get the data cursor of MATLAB ${ }^{\circledR}$ to pick exactly on the thrust value of $64.23 \mathrm{~N}$ on both sides of the curve hence, we have what is displayed in Fig. 3. The corresponding value for $64.23 \mathrm{~N}$ on the right-hand-side of the curve is 10.25 seconds. The data cursor of MATLAB kept jumping away from this value because on the .csv file this point does not exist. Since we are interested in just the time at which the curve reaches $64.23 \mathrm{~N}$ on both sides we made-do with the choice depicted in Fig. 3. Aside, the difference between $10.25 \mathrm{~s}$ and $10.03 \mathrm{~s}$ is $0.22 \mathrm{~s}$, this value is negligible!

\section{Average Thrust}

Average thrust is the average instantaneous force the motor produces during its burn and is measured in Newton $(\mathrm{N})$. Note that because most thrust curves of SRM are not flat, most of the time the motor is not producing the average thrust. The average thrust tells you how heavy a rocket the motor can lift, although since different motors produce different shaped thrust curves, it can be misleading. We use the following code to compute the average thrust for this SRM test result:

$$
\text { avg_thrust_1 = mean(f2) }
$$

The average thrust obtained, is then used to compute thrust-to-weight as given in (1). This dimensionless value is used to determine design lift-off mass of rockets. For sounding rockets, typical values for thrust-to-weight ratio is within the range of 3-4 (Taylor, 2009):

$T_{w}=\frac{\text { Thrust }_{\text {avg }}}{\text { Weight }}=\frac{705.3}{9.8 \times m_{L}}$,

where, $m_{\mathrm{L}}$ is the lift off mass of the rocket. Note, (1) must be greater than 1 for the rocket to lift hence, we can simulate for various values as depicted in Fig. 4. Hence, one tailor a particular sounding rocket design to attain a pre-determined altitude.

From Fig. 4, the range for thrust-to-weight 1.2-3 is chosen as the feasibly design region based on the existing technology on ground in CSTP at the time of this writeup. $T_{\mathrm{w}}$ higher than 3 will require a special composite material to be designed for the combustion chamber and airframe of the rocket such that the lift-off mass be less than $24 \mathrm{~kg}$. While a lift-off mass of $60 \mathrm{~kg}$ will result to a $T_{\mathrm{w}}$ of just 1.2, though this means the rocket will lift-off but might not attain a very high altitude.

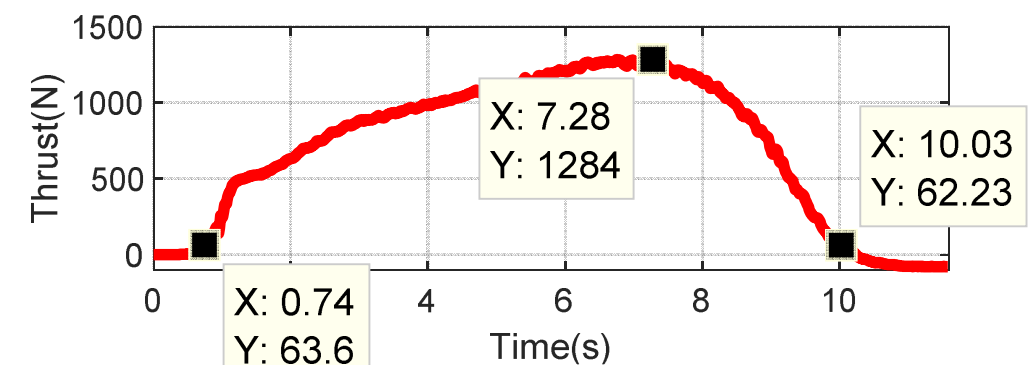

Fig. 3: Time against thrust profile of the tested SRM

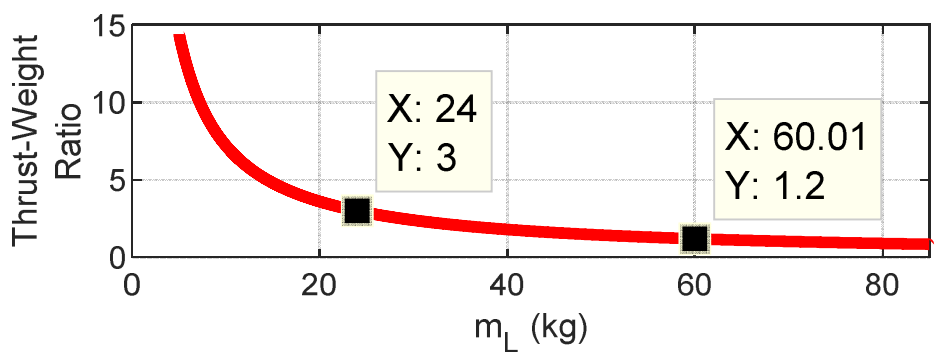

Fig. 4: Thrust-weight ratio curve 


\section{Maximum Thrust}

The Maximum thrust is the maximum amount of force produced by the motor during its burn. Like average thrust, this is measured in Newton (N). Generally, there is a small spike near the beginning which has the highest thrust, although the shape of the curve varies with the motor design and propellant mixture. For the SRM under analysis in this study, the value of maximum thrust was computed by using the following MATLAB ${ }^{\circledR}$ command:

max_thrust $=\max (f 2)$

\section{Total Impulse}

Total Impulse, $I_{\mathrm{T}}$ is equal to integral of thrust over the time of operation of the engine. This is the product of thrust times duration over the motor burn time and is measured in Newton seconds (Ns). This measures the total amount of momentum imparted to the rocket by the motor. The total impulse (along with such factors as rocket mass and air drag) determines how high the motor can propel your rocket. The impulse class is a letter code assigned to a range of total impulse with each letter being twice as powerful as the previous. A typical motor example is Cesaroni Pro38 266H125-12. H is the impulse range, 125 is the average thrust $(\mathrm{N})$ and 12 seconds is the motor delay-from motor burnout to ejection, 266 is the total impulse (Ns). The motor manufacture's name is Cesaroni and 38 is the motor diameter in millimeters (NAR, 2018). Such Long delays (12 sec) are best for lighter rockets, which will coast upwards for a long time. Heavier rockets usually do better with shorter delays (typical of which is 3-4 seconds)-otherwise the rocket might fall back down to the ground during the delay time. Motors marked with a time delay of 0 are booster engines. They are not designed to activate recovery systems. They are intended for use as lower-stage engines in multi-stage rockets. They are designed to ignite the next stage engine immediately once their own thrust is finished. Often their labels are printed in a different color to help prevent you from using them in a typical rocket. In a multi-stage rocket, you would usually select a very long delay for your topmost engine. Letter classifications of rocket motors refer to the range of total impulse, not to the average or maximum thrust as depicted in Table 1. Hence, we computed total impulses for the SRM in this study using the MATLAB ${ }^{\circledR}$ code:

$$
\text { imp_tot }=\text { avg_thrust } * t \_b
$$

\section{Specific Impulse}

The efficiency of a rocket is defined by specific impulse defined as given in (2). Specific impulse is used for all types of rocket (chemical, electric and for ramjet/scramjet) specific impulse can be considered as the inverse of specific consumption. The higher the specific impulse, the better is the rocket (Kuentzmann, 2002):

$$
I_{s}=\frac{F}{\dot{m} g_{o}}
$$

where, $I_{s}$ is the specific impulse (s), $\dot{m}$ is the mass flow rate ejected by the rocket $\left(\mathrm{kg}_{\mathrm{s}} \mathrm{s}^{-1}\right), \mathrm{g}_{0}$ is the reference gravity $\left(9.806 \mathrm{~m} \cdot \mathrm{s}^{-2}\right)$.

In literature (Atwood et al., 2013), the gas generation rate from the surface of the propellant during combustion is described by (3):

$$
\dot{m}=\rho_{p} A_{b} \dot{r}
$$

where, $\rho_{g}=$ is the propellant mass, $A_{\mathrm{b}}=$ area of the burning surface, $\dot{r}=$ surface regression speed or burn rate.

From (3), we can write the following expression:

$$
\dot{m}=\left\{\frac{m_{p}}{v_{p}}\right\} A_{b} \dot{r}=\left\{\frac{m_{p}}{A_{b} l_{p}}\right\} A_{b} \dot{r}=\frac{m_{p}}{l_{p}} \dot{r}=m_{p}\left\{\frac{\dot{r}}{l_{p}}\right\}
$$

where, $v_{p}=$ propellant volume and $l_{p}$ is the length of the propellant grain.

Note, the length of the propellant grain regresses during combustion. Meaning, not only is the grain area depleting but the entire volume! Hence, it could be seen as an entity embedded with the burn rate of the propellant. Hence we can re-write (4) as:

$\dot{m}=m_{p} \dot{r}$

Bear in mind that burn rate is define as"

$\dot{r}=a P_{c}^{n}$

where, $a$ is the burn rate coefficient, $n$ is the pressure exponent and $P_{c}$ is the combustion chamber pressure. The values of $a$ and $n$ are determined empirically for a particular propellant formulation and cannot be theoretically predicted. It is important to realize that a single set of $a, n$ values are typically valid over a distinct pressure range. More than one set may be necessary to accurately represent the full pressure regime of interest (Davenas, 1993). This means both pressure and temperature are not constant in the SRM. Observe if you will, that mass flow rate of a fluid is established based on a constant volume, pressure and temperature, White (2002). Mathematically, for a rocket nozzle, the mass flowrate (Crowe, 2009) is defined as:

$$
\dot{m}=0.65 \frac{P_{c} A_{t}}{\sqrt{R T_{c}}},
$$

where, $R$ is the gas constant of the gases in the chamber and $A_{\mathrm{t}}$ is the throat area of the nozzle. 


\begin{tabular}{llc}
\multicolumn{2}{l}{ Table 1: SRM Classification } & \\
\hline Class & Impulse class & Category \\
\hline $\mathrm{H}$ & $160.01 \mathrm{Ns}$ to $320.01 \mathrm{Ns}$ & Level 1 \\
$\mathrm{I}$ & $320.01 \mathrm{Ns}$ to $640.00 \mathrm{Ns}$ & \\
$\mathrm{J}$ & $640.01 \mathrm{Ns}$ to $1,280.00 \mathrm{Ns}$ & Level 2 \\
$\mathrm{~K}$ & $1,280.01 \mathrm{Ns}$ to $2,560.00 \mathrm{Ns}$ & \\
$\mathrm{L}$ & $2,560 \mathrm{Ns}$ to $5,120.00 \mathrm{Ns}$ & \\
$\mathrm{M}$ & $5,120.00 \mathrm{Ns}$ to $10,240.00 \mathrm{Ns}$ & Level 3 \\
$\mathrm{~N}$ & $10,240.00 \mathrm{Ns}$ to $20,480.00 \mathrm{Ns}$ & \\
$\mathrm{O}$ & $20,480.00 \mathrm{Ns}$ to $40,960.00 \mathrm{Ns}$ & \\
\hline
\end{tabular}

Both (5) and (7) will give a constant value as mass flow rate. This does not appeal to us. Here, we want to account for the rate at which the propellant mass depletes during the SRM operation. Hence, from (5), it can be said that an exponential function is being multiplied with the mass of the propellant. One should expect also that the exponential function be negative, since this will mean the mass of the propellant will be decreasing all through the burn-time. With this understanding and ignoring the complex effect of pressure, temperature and other factors, we simply model the depleting propellant mass (mass flow rate inside the combustion chamber) during combustion in a SRM as:

$\dot{m}=m_{p}^{(-\zeta \delta)}$

where, $\zeta$ is a constant selected based on the residual propellant mass in the combustion chamber after burntime). We then substituted the mass flow rate in (2) with (8) and simulation of (8) is shown in Fig. 4 and the following MATLAB code was used to achieve the required simulation:

$t b 1=(0: 0.01: 9.29)$

$t b=$ linspace $(0,1,930)$

$m p=10.6$

figure(); hold on

for zeta $=\left[\begin{array}{lll}3 & 3.7 & 4.2\end{array}\right]$

dec_mass $=m p * \exp (-z e t a * t b)$

dec_m $=$ dec_mass'

plot $(t b 1$, dec_m)

grid

end

hold off

Notice that from Fig. 4, a residue of propellant mass for three values of $\zeta$ is displayed. These values were picked randomly to match the quantity of propellant left in the chamber after the test. It can be clearly seen from Fig. 5 , that (8) can be used effectively to describe the mass flowrate of the propellant inside the chamber of SRM provided one knows the mass of the propellant residue after motor operation. Notice also that as $\zeta$ increases from 3 to 4.2 , the residual mass decreases. Hence we proceed to compute the specific impulse of the motor with the following code added to the previous ones:

$$
\begin{aligned}
& g=9.82 \\
& \left.I s p \_1=t \text { _impulse/(dec_m }{ }^{*} g\right)
\end{aligned}
$$

The second approach we use to compute the specific impulse was to use the constant value of the propellant mass $(10.6 \mathrm{~kg})$. Both approaches gave us the same answer. Though, the second approach gave an answer in an array with the first cell as $63.01 \mathrm{~s}$ and the rest zeros.

\section{Chamber Pressure}

From physics, pressure is defined as the product of force and area as given in (9). Here, we introduce the pseudo-numerical approach to determine this pressure for chamber:

$$
P_{c}=\text { Thrust } \times\left(A_{b}\right)^{-1}
$$

where, the thrust is obtained from the test and $A_{\mathrm{b}}$ is assumed to be equivalent to the total surface area of a hollow cylinder deduced as given in Fig. 5 .

Using the experimental values of the force $(f 2)$ and the total surface of the hallow grain propellant depleting modeled as given in (10). We assume that the rate of exponential decay in (10) is same as that in (8) hence, we can write:

$$
\begin{aligned}
& A_{b}=\left\{2 \pi r_{p} l_{p}+2 \pi R_{p} l_{p}+2\left(\pi R_{p}^{2}-\pi r_{p}^{2}\right)\right\} e^{(-\zeta \delta)} \\
& =0.6295 e^{(-\zeta \delta)}
\end{aligned}
$$

where, $r$ is the internal radius, $R$ is the external radius, $l$ is the length of the cylindrical shapes propellant grain as depicted in Fig. 6:

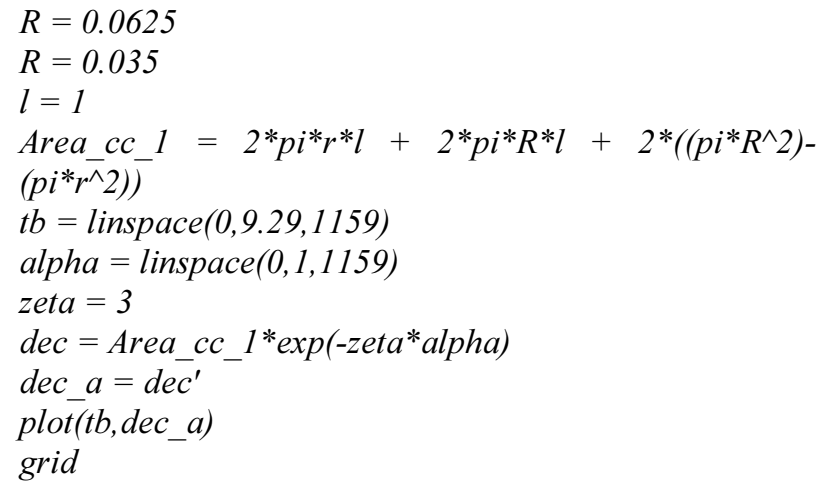

Notice the similarity in trend between Fig. 5 and 7, this is expected. The difference comes only at the $y$ intercepts of both graphs. We don't expect anything outside what we have due the fact that one can relate that the total surface area of the hallow cylindrical propellant grain has the same dynamics as the mass of the propellant inside the chamber. 


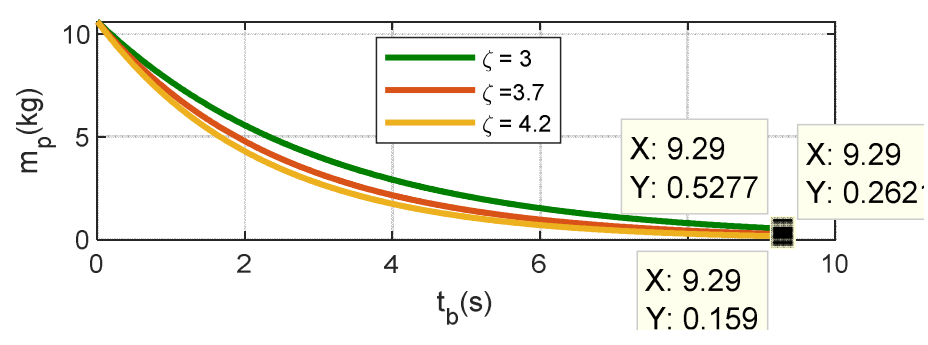

Fig. 5: Mass flowrate of propellant inside the combustion chamber of SRM

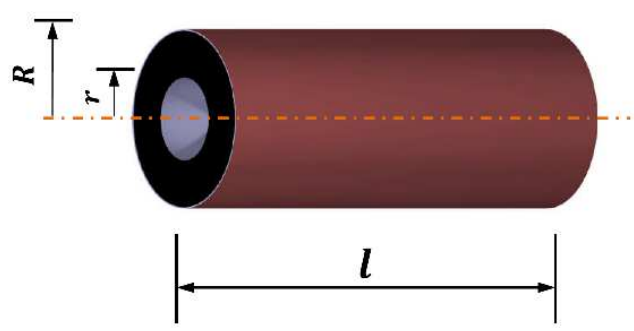

Fig. 6: Hallow cylindrical propellant grain as it seats in the SRM

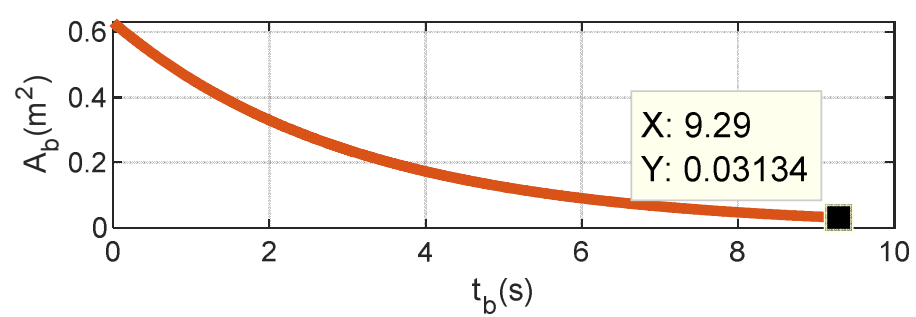

Fig. 7: Simulation of depleting propellant surface area during combustion

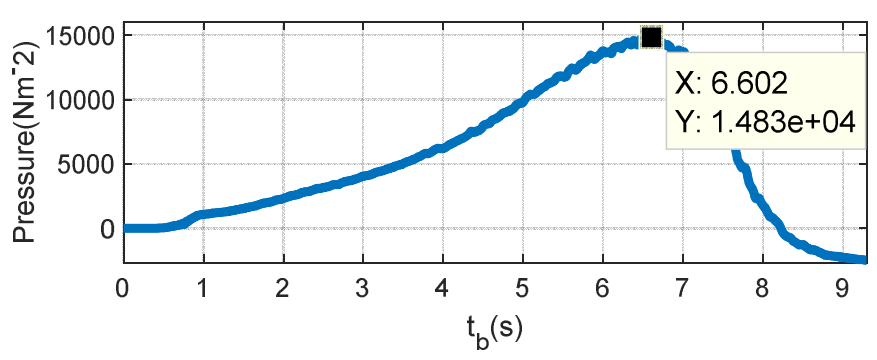

(a)

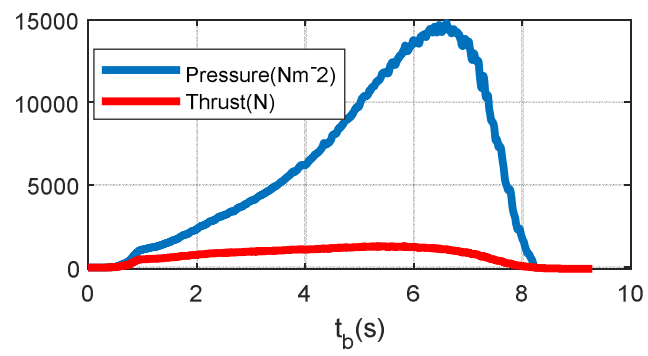

(b)

Fig. 8: Pressure and thrust of SRM

Computing SRM chamber pressure pseudo-numerically with (9) gave the result in Fig. 8a. A combined thrust and pressure in the combustion chamber is given in Fig. $8 \mathrm{~b}$ for close perusal. It can be seen that both maximum thrust (7.28, 1,284, from Fig. 3) and pressure $\left(6.602,1.483 \times 10^{4}\right.$, from Fig. 8a) occurred at about 0.68 seconds apart (7.286.60s). Typically, the trend in Fig. $8 \mathrm{~b}$ observed to be similar with those in some selected independent studies (Singh, 2013; Sieder-Katzmann et al., 2017; Fedaravičius et al., 2015; Chankapoe et al., 2013): pr_1 $=f 2 . /$ dec $a$;

max $\_$pre_1 $=\overline{\max }\left(\mathrm{pr} \_1\right)$

Avg_pre_l $=\operatorname{mean}\left(p r_{-} 1\right)$

figure(1)

$\operatorname{plot}(t b, f 2)$

The above MATLAB ${ }^{\circledR}$ code used for generating the result in Fig. 7 was also used to compute the average chamber pressure.

The need for comparison between the pseudonumerically computed SRM chamber pressure as 
presented in this study, with literature-based exact solution is of utmost importance.

\section{Exact Solution to Chamber Pressure}

Chamber pressure of solid rocket propellant is governed by a nonlinear first order ordinary deferential equation (Cantwell, 2010), given as:

$\frac{d P_{t 2}}{d t}+\left(\frac{1}{\tau}\right) P_{t 2}-\beta\left(P_{t 2}\right)^{n}=0$

where, the characteristic time is:

$\tau=\frac{\left(\frac{\gamma+1}{2}\right)^{\frac{\gamma+1}{2(\gamma-1)}}}{\left(\gamma R T_{t 2}\right)^{1 / 2}}\left(\frac{V}{A^{*}}\right)$,

The SRM has the character of a Helmholtz resonator and the inverse of (12) is the natural 'Coke

Bottle' frequency of the rocket motor. The constant in the nonlinear term of (11) is:

$\beta=\left(\frac{K\left(\rho_{p}-\rho_{g}\right) A_{b}}{T_{1}-T_{p}}\left(\frac{R T_{t 2}}{V}\right)\right)$

where:

$\rho_{p}=$ Solid propellant density

$\rho_{g}=$ Gas density

$V=$ The chamber volume

$A_{\mathrm{b}}=$ Area of burning surface

$T_{1}=$ imperial detonation temperature

$T_{\mathrm{p}}=$ Propellant temperature

$K=$ Imperial constant for a given propellant

$T_{\mathrm{t} 2}=$ Chamber designated temperature

$R=$ Idea gas constant

$A^{*}=$ Throat area

$n$ = Imperial exponent, approximately independent of temperature

Let's determine the exact integral of this equation and compare the behavior of the system with the linearized solution for both $n<1$ and $n>1$. It can be shown that the steady state solution to (11) which the time derivative term is zero is:

$$
\overline{P_{t 2}}=(\tau \beta)^{\frac{1}{1-n}}
$$

Let:

$$
H=\frac{P_{t 2}}{\overline{P_{t 2}}}
$$

$\eta=\frac{t-t_{0}}{\tau}$

Inserting (15) and (16) in (11) we get:

$\frac{d H}{d \eta}=H^{n}-H$

Rearranging (17) we get:

$\frac{d H}{H^{n}-H}=d \eta$

Integrating (18) gives:

$\frac{1-H^{1-n}}{1-H_{0}^{1-n}}=e^{-(1-n) \eta}$

where, $H_{0}$ is the initial value of $P_{t 2} / \overline{P_{t 2}}$ and the initial value of $\eta$ is taken to be zero. Now solving for $H$ we get:

$H=\left(1-\left(1-H_{0}^{1-n}\right) e^{-(1-n) \eta}\right)^{\frac{1}{1-n}}$

Several cases of the solution of (20) are shown in Fig. 9. The exact solution shows that if $n>1$ there is no actual steady state, the chamber pressure either decays to zero or blows up. If $n<1$ then the chamber pressure will return to the steady state value even in the face of a large deviation away from steady state.

Comparing Fig. 8a with Fig. 9, the pseudo-numerical SRM pressure at the initial stage of the motor operation is a combination of the exact solution for which $n=1.2$ i.e., $n>1$. Hence, the pressure kept rising for $t_{0}=0 \mathrm{~s}$ to $t=$ $6.62 \mathrm{~s}$. At $t=6.62 \mathrm{~s}$, the SRM pseudo-numerical pressure began to decay $(n>1)$ until it got to zero at the end of the burn time. For us, since the SRM did not explode or show any physical sign of damage beyond re-use, the pseudonumerical pressure could be associated with an $n$ value greater than 1 but less than 1.2. The maximum pseudonumeric pressure deduced is of important, as it will guide in future light-weight material selection for combustion chamber design. Table 2 below gives all the performance characteristics of the SRM considered in this study.

Just like the maximum pressure, the maximum thrust informs of the possible yield point stress of the material that should be chosen for the combustion chamber. Base on the characteristics of the SRM depicted in Table 2, the motor tested in this study is designated Pro130 6552CSTP M705-3. The major reason for the 3 seconds delay associated with the motor stems from the fact that the final mass (no propellant) of the rocket is heavy. We will not want a situation where the rocket with Prol30 6552CSTP M705-3 to start descending before parachute eject takes place. 


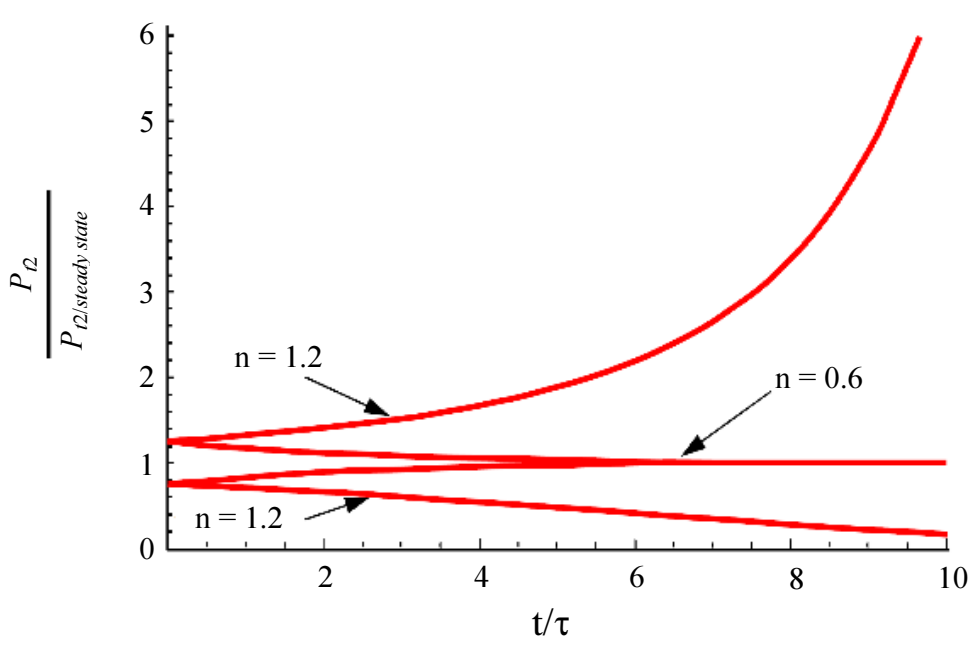

Fig. 9: Exact solution to SRM pressure

Table 2: Parameters of the SRM tested

\begin{tabular}{lll}
\hline $\mathrm{S} / \mathrm{N}$ & Parameters & Values \\
\hline 1 & Propellant mas, $m_{p}(\mathrm{~kg})$ & 10.6 \\
2 & Propellant residue mass, $m_{\mathrm{pr}}$ & 0.533 \\
3 & Burn time, $t_{b}(\mathrm{~s})$ & 9.29 \\
4 & Max. Thrust $(\mathrm{N})$ & $1.28 \times 10^{3}$ \\
5 & Total Impulse $(\mathrm{Ns})$ & $6.552 .6 \times 10^{3}$ \\
6 & Specific Impulse (s) & 63.01 \\
7 & Avg. Thrust (N) & 705.34 \\
8 & Max. Chamber pressure $\left(\mathrm{Nm}^{-2}\right)$ & $14 \times 10^{3}$ \\
9 & Average Chamber pressure $\left(\mathrm{Nm}^{-2}\right)$ & $5.5 \times 10^{3}$ \\
10 & Chamber Diameter $(\mathrm{m})$ & 0.125 \\
11 & Throat diameter $(\mathrm{m})$ & 0.025 \\
12 & Nozzle exit diameter $(\mathrm{m})$ & 0.09 \\
13 & Propellant Grain Area $\left(\mathrm{m}^{2}\right)$ & 0.629 \\
14 & Propellant web thickness $(\mathrm{m})$ & 0.0275 \\
15 & Propellant Grain length $(\mathrm{m})$ & 1 \\
\hline
\end{tabular}

\section{Conclusion}

To characterize a SRM, it was tested on a static test rig. Thrust with time values obtained from the test were imported into MATLAB ${ }^{\circledR}$ for analysis and computations. Pressure of the SRM chamber was obtained in relation with the experimental thrust values multiplied by the reciprocal of the propellant grain area. After defining the mass flow rate inside the combustion chamber in a novel mathematical model, the propellant grain area was also modelled as a hollow cylinder taking a cue from the novel propellant mass model. These novel methods of accounting for the propellant mass and grain area dynamics in the combustion chamber, in combination with experimental thrust values used empirically to deduce chamber pressure, necessitated the coining of the phrase pseudo-numerical. Thus, specific impulse and chamber pressure of the SRM were computed using the pseudo-numerical approach.

\section{Future Work}

There is the need to estimate the temperature of the combustion chamber of the SRM using the pseudonumerical approach. This will also help in material selection for the combustion chamber. Experimental values for pressure in the combustion chamber should also be used to compare with the pseudo-numerical approach suggested here.

\section{Acknowledgment}

Authors wish to acknowledge the relentless effort of staff memebers at CSTP, especially those working at the system dynamics laboratory.

\section{Author's Contributions}

Aliyu Bhar Kisabo: Designed the study, and drafted the first manuscript.

Ngozi Nwokolo and Funmilayo Adebimpe Aliyu: Verified all result and computer simulations and assisted with litereture search.

Sholiyi Olusegun Samuel: Supervised the entire study and edited the final manuscript.

\section{Ethics}

Authors declare that there are no ethical issues that may arise after the publication of this manuscript. This article is original and contains unpublished material.

\section{References}

Abdel, A.R., M. Mohamed, H. Abdalla and M. Elsenbawi, 2015. Analytical prediction of dual-thrust rocket motors under uncertainties. Proceedings of the 16th International Conference on Aerospace, Sciences and Aviation Technology, (SAT' 15). 
Aliyu, B.K., C.A. Osheku, E.O. Oyedeji, M.A.L. Adetoro and A.A. Okon et al., 2015. Validating a novel theoretical expression for burn time and average thrust in solid rocket motor design. Adv. Res., 5: 1-11, 2015. DOI: 10.9734/AIR/2015/18468

Atwood, A.I., K.P. Ford and C.J. Wheeler, 2013. Highpressure burning rate studies of solid rocket propellants. Progress Propuls. Phys., 4: 3-14. DOI: $10.1051 /$ eucass/201304003.

Cantwell, B.J., 2010. Aircraft and rocket propulsion. AIAA Educational Series. Sandford University.

Chankapoe, S., N. Winya and N. Pittayaprasertku, 2013. Performance investigation of solid-rocket motor with nozzle throat erosion. Int. J. Mech. Aerospace Indus. Mechatron. Eng.

Crowe, C.T., 2009. Engineering Fluid Mechanics. 9th Edn., John Wiley and Sons, New Jersey, ISBN-13: 978-0470-25977-1.

Davenas, A., 1993. Solid Rocket Propulsion Technology. 1st Edn., Elsevier Science and Technology Books, New York, ISBN-10: 0080409997, pp: 606.

Fedaravičius, A., S. Rackauskas, A. Survila and L. Patašiene, 2015. Design of the testing system for solid propellant rocket motor thrust measurements using mathematical modelling techniques. J. Measurements Eng., 3: 123-131.
Kuentzmann, P., 2002. Introduction to solid rocket propulsion. Office National d'Etudes et de Recherches Aérospatiales 29, avenue de la Division Leclerc - BP 7292322 Châtillon Cedex, France.

MPDO, 2018. Motor Performance Data Online. http://www.thrustcurve.org/motorstats.shtml

NAR, 2018. National Association of rocketry. http://www.nar.org/standards-and-testingcommittee/standard-motor-codes/

Sieder-Katzmann, J., C. Bach, K. Kleebusch and M. Tajmar, 2017. Development history and verification of the flight model of a $500 \mathrm{~N}$ ethanol/LOX rocket engine. Proceedings of the 7th European Conference for Aeronautics and Aerospace Sciences, (AAS' 17), At Milano.

Singh, S., 2013. Solid rocket motor for experimental sounding rockets. Adv. Aerospace Sci. Applic., 3: 199-208. http://www.ripublication.com/aasa.htm

Taylor, T.S., 2009. Introduction to Rocket Science and Engineering. 1st Edn., CRC Press, New York, ISBN-10: 1420075284, pp: 324.

Turner, M.J.L., 2006. Rocket and Spacecraft Propulsion: Principles, Practice and New Developments. 2nd Edn., Springer, New York, ISBN 3540270418, pp: 314.

White, F.M., 2002. Fluid Mechanics. 5th Edn., McGrawHill Series in Mechanical Engineering, ISBN-13: 978-0072402172. 